\title{
Baicalin induced dendritic cell apoptosis in vitro
}

\author{
Huahua Zhang ${ }^{1,2+}$, Qingqing Jiao ${ }^{3+}$, Qianfeng Gong ${ }^{2}$, Yan Zhang ${ }^{1}$, Weidong Zhang ${ }^{1}$ and Zhenlin $\mathrm{Hu}^{1}$ * \\ 'School of Pharmacy, Second Military Medical University, Shanghai, China \\ 2 Department of Traditional Chinese Medicine, Jiangxi University of Traditional Chinese Medicine, Nanchang, China \\ 3 Xinhua Hospital, School of Medicine, Shanghai Jiao Tong University, Shanghai, China
}

\section{Edited by:}

Tong-Hui Ma, Jilin University, China

\section{Reviewed by:}

Ai-Jun Liu, The Second Military Medical University, China

Hong Yang, Liaoning Normal University, China

Shailendra Shivaji Gurav, Pune District Education Association's Seth Govind Raghunath Sable College of Pharmacy, India

\section{${ }^{*}$ Correspondence:}

Zhenlin Hu, School of Pharmacy,

Second Military Medical University,

Guohe Road 325, Shanghai 200433,

China.

e-mail: zhenlinhu@hotmail.com

${ }^{+}$Huahua Zhang and Qingqing Jiao have contributed equally to this work.
This study was aimed to investigate the effects of baicalin (BA), a major flavonoid constituent found in the herb Baikal skullcap, on dendritic cells (DCs). DCs were generated by culturing murine bone marrow (BM) cells for 6 days with granulocyte-macrophage colony-stimulating factor and interleukin (IL)-4, and lipopolysaccharide (LPS) was added on day 5 to stimulate DCs maturation. The expression levels of DC maturity markers (CD80/CD86) were assessed by flow cytometry using direct immunofluorescence method. IL-12 levels in the culture supernatants were assayed by ELISA. Apoptosis of DCs was analyzed by flow cytometry after annexin V/ propidium iodide staining. The mitochondrial membrane potential $\left(\Delta \psi_{\mathrm{m}}\right)$ changes were measured by using the J-aggregate forming lipophilic cation 5, 5',6,6'-tetrachloro-1, 1',3,3'-tetraethylbenzimi dazolcarbocyanine iodide (JC-1). Exposure of DCs to BA (2-50 $\mu \mathrm{M})$-during BM cell differentiation showed no effects on the up-regulation of CD80/CD86 expression on DCs in response to LPS stimulation, but reduced DCs recovery by inducing apoptosis, and significantly inhibited the release of IL-12 to culture supernatants. BA-induced DC apoptosisin a time-and dose-dependent way, and immature DCs were more sensitive for BA-induced apoptosis than mature DC. BA also induced $\Delta \psi_{m}$ changes in DCs. These results demonstrate that BA induces selective apoptosis in immature DCs possibly through mitochondria-mediated pathway.

\section{INTRODUCTION}

Baicalin (BA) is a major flavonoid constituent (Figure 1) found in the traditional Chinese medicinal herb Baikal skullcap (Scutellaria baicalensis Georgi), known as "Huang gin" in China and "Ogon" in Japan, which has been widely used for the treatment of various diseases such as pneumonia, hepatitis, and diarrhea (Huang et al., 1986). Previous studies have demonstrated that BA possesses a wide range of pharmacological and brological activities such as anti-inflammatory, anti-allergic, antimicrobial, antioxidant, and anti-tumor properties (Zhang et al., 2003). Its anti-inflammatory activity has been estimated in various animal models of acuteand chronic inflammation (Kubo et al., 1984; Lin and Shieh, 1996; Zeng etal. 2007). Evidence shows that its anti-inflammatory actions are associated with the suppression of functions of various inflammatory cells and inhibition the production of pro-inflammatory mediators such as TNF- $\alpha$, interleukin (IL)-1, and PGE (Chung et al., 1995; Krakauer et al., 2001). Recently, it is reported that BA can inhibit the proliferation of mouse T-lymphocytes stimulated with various mitogens and arrest lymphocytes in $G_{0} / G_{1}$ phase (Li et al., 2009). The results of these studies indicate that BA may have pronounced immunoregulatory properties, although the underlying mechanisms still remain to be fully elucidated.

Dendritic cells (DCs) are potent antigen-presenting cells that initiate lymphocyte activation (Banchereau and Steinman, 1998; Banchereau et al., 2000). They develop from BM precursors and then migrate via the bloodstream to almost every tissue, where they reside as immature DCs. During pathogen invasion, or after xposure to inflammatory mediators, DCs undergo phenotypic and functional maturation, a state characterized by the upregulation of class II major histocompatibility complex (MHC II) and costimulatory molecules (CD80/CD86) and the production of cytokines such as IL-12. Upon maturation, DCs in tissues migrate into afferent lymphatics and move to the $\mathrm{T}$ cell areas of lymph nodes, where they encounter naive $\mathrm{T}$ cells and initiate adaptive immune responses (Butcher and Picker, 1996). The apoptosis of DCs, upon completion of their task of antigen presentation, appears to serve as a negative immunoregulatory mechanism that may be crucial in controlling the magnitude of immune reactions against a given antigen (Hildeman et al., 2007). It has been demonstrated that elimination of DCs in experimental animals leads to immunologic ignorance or even paralysis of antigen-specific $\mathrm{T}$ cells after antigen exposure (Jung et al., 2002). Accordingly, the induction of premature DC death by immunomodulatory drugs appears to be a major pharmacologic principle of antiinflammatory treatments (Hackstein and Thomson, 2004). In contrast, defects in DC apoptosis lead to DC accumulation, and through chronic lymphocyte activation, the development of autoimmunity (Chen et al., 2006).

The anti-inflammation effects of BA have been well-established and accumulated evidence indicates that BA may have potential immunomodulatory properties (Kubo et al., 1984; Chung et al., 1995; Lin and Shieh, 1996; Krakauer et al., 2001; Zhang et al., 2003; Zeng et al., 2007; Li et al., 2009). However, it effects on DCs have not been addressed. In this study, we used murine BM-derived DCs (BMDCs) to analyze its direct effects on DCs. 


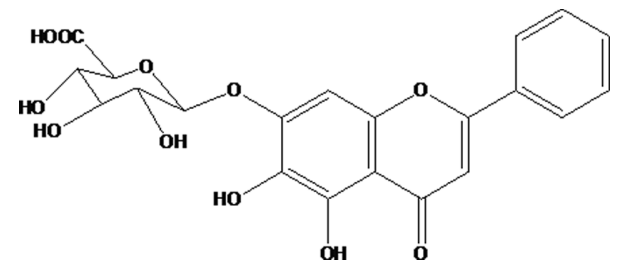

FIGURE 1 |The chemical structure of BA.

\section{MATERIALS AND METHODS REAGENTS}

Baicalin (purity $>99 \%$ ) was purchased from National Institute for the Control of Pharmaceutical and Biological Products, China. Lipopolysaccharide (LPS) and RPMI-1640 were purchased from Sigma (St Louis, MO, USA). Fetal calf serum was purchased from GIBCO-BRL (Gland Island, NY, USA). Recombinant mouse granulocyte-macrophage colony-stimulating factor (GM-CSF) and IL-4 were purchased from R\&D System (Minneapolis, MN, USA). Fluorescein-5-isothiocyanate (FITC)-anti-mouse CD11c, PE-antimouse CD80, PE-Cy5-anti-mouse CD86, and anti-murine Fc receptor monoclonal antibodies were purchased from PharMingen (San Diego, CA, USA). The J-aggregate forming lipophilic cation 5, $5^{\prime}, 6,6^{\prime}$-tetrachloro-1, 1' $, 3,3^{\prime}$-tetraethylbenzimidazolcarbocyan ine iodide (JC-1) was purchased from Molecular Probes (Eugene, OR, USA). Annexin V-FITC Apoptosis Detection kit was purchased from Keygen Biotech. Co., Ltd (Nanjing, China). All other chemicals used were of the highest grade available commercially.

\section{CULTURE OF BONE MARROW-DERIVED DCS}

Bone marrow-derived DCs were generated according to the method described previously (Inaba et al., 1992) with some modification. In brief, BM cells were flushed from the femurs and tibiae of female C57BL/6 mice (purchased from Shanghai SLAC Laboratory Anlmal Co., Ltd, Shanghai, China), filtered through a Falcon 100- $\mu$ m nylon cell strainer (BD Labware), and depletêd of red bloodeells by $5 \mathrm{~min}$ incubation in ACK lysis buffer $\left(0.15 \mathrm{MNH}_{4} \mathrm{Cl}, 1.0 \mathrm{mM} \mathrm{KHCO}_{3}\right.$, $0.1 \mathrm{mM} \mathrm{Na} 2$ EDTA, $\mathrm{pH}$ 7.4). Whole BM cells were plated in six-well plates (BD Labware) in RPMI-1640 supplemented with $10 \% \mathrm{FCS}$, GM-CSF $(10 \mathrm{ng} / \mathrm{ml})$, and $\mathrm{LL}-4(10 \mathrm{ng} / \mathrm{ml})$, and incubated at $37^{\circ} \mathrm{C}$ and $5 \% \mathrm{CO}_{2}$. Three days later, the floating cells (mostly granulocytes) were removed, and the adherent cells were replenished with fresh medium containing GM-CSF and IL-4. Non-adherent and loosely adherent cells were harvested on day 6 as immature DC (typically contained $>90 \%$ cells expressing CD11c and MHC II on the surface, as determined by flow cytometry). To stimulate DCs maturation, LPS ( $500 \mathrm{ng} / \mathrm{ml}$ ) was added to the culture on day 5 as indicated. For analysis of BA effects on DC development, BA $(2-50 \mu \mathrm{M})$ was added on day 3 .

\section{PHENOTYPIC MARKER ANALYSIS}

Dendritic cells harvested on day 6 were washed and suspended in fluorescence-activated cell-sorting analysis (FACS) Buffer (phosphate buffered saline containing $0.1 \%$ bovine serum albumin and $0.01 \% \mathrm{NaN}_{3}$ ), and stained with the fluorescence-conjugated monoclonal antibodies recognizing $\mathrm{CD} 11 \mathrm{c}, \mathrm{CD} 80$, or CD86 in the presence of anti-murine Fc receptor monoclonal antibody as described previously (Zhang et al., 2004). Samples were analyzed by flow cytometry on a FACSCalibur (Becton Dickinson, USA) with the CellQuest software package.

\section{IL-12 ASSAY}

Interleukin-12 levels in the supernatants of BMDCs were determined by ELISA kits (Bender MedSystems, Vienna, Austria), according to the manufacturer's instructions.

\section{DETECTION OF APOPTOSIS}

The annexin V-FITC binding and propidium iodide (PI) staining assay were used to assess apoptosis of DCs as described previously (Koopman et al., 1994). $1 \times 10^{6}$ cells were stained with the annexin V-FITC and PI provided with the Annexin V-FITC Apoptosis Detection kit (purchased from Keygen Biotech. Co., Ltd, Nanjing, China), according to the manufacturer's instructions. Stained cells were analyzed via a FACSCalibur and the CellQuest software (Becton Dickinson, USA).Annexin V positive cells were determined as apoptosis cell

\section{ASSAY FOR MITOCHONDRIAL MEMBBRANE POTENTIAL}

Mitochondrial membrane potential $\left(\Delta \psi_{\mathrm{m}}\right)$ was determined by flow cytometry using J-aggregate forming lipophilic cationic probe JC-1 $\left(5,5^{\prime}, 6,6^{\prime}\right.$-tetrachloro-1, 1',3,3'-tetraethylbenzimida zolcarbocyanine iodide) following the manufacturer's protocol (Molecular Probes, Eugene, OR, USA), and as described previously (Cossarizza et al., 1993). In brief, JC-1 was dissolved in dimethyl sulfoxide $(5 \mathrm{mg} / \mathrm{ml})$, and $50 \mu \mathrm{l}$ were added to $10 \mathrm{ml}$ of medium. $5 \times 10^{5}$ DCs were incubated with $2 \mathrm{ml}$ of medium containing JC- 1 for $15 \mathrm{~min}$ at $37^{\circ} \mathrm{C}$ and then washed twice with PBS. Cells were then resuspended in $0.5 \mathrm{ml}$ of PBS and analyzed on a FACSCalibur (Becton Dickinson, USA) to detect green fluorescence at excitation/emission wavelengths of 485/530 nm and red fluorescence at excitation/emission wavelengths of $550 / 595 \mathrm{~nm}$. Since JC-1 exhibits potential-dependent accumulation in mitochondria, indicated by a fluorescence emission shift from green $(\sim 525 \mathrm{~nm})$ to red $(\sim 590 \mathrm{~nm})$, mitochondrial depolarization is indicated by a decrease in the red-to-green fluorescence-intensity ratio.

\section{STATISTICS}

All data were expressed as the mean values \pm SD. Differences between groups were examined for statistical significance using the Student's $t$-test. A value of $p<0.05$ was considered statistically significant.

\section{RESULTS}

\section{EFFECTS OF BA ON DC MATURATION AND APOPTOSIS}

The effect of BA on DCs was initially investigated in BM cell cultured for 6 days with GM-CSF and IL-4, which generates mainly BMDCs. BA $(0-50 \mu \mathrm{M})$ was added on day 3 , and LPS $(500 \mathrm{ng} / \mathrm{ml})$ was added on day 5 to stimulate DC maturation. The surface expressions of CD11c, CD80, and CD86 on BA-treated and -untreated DCs were examined by flow cytometry, and levels of IL-12 in the supernatants were measured by ELISA. The results demonstrated that more than $90 \%$ of non-adherent and loosely adherent cells 
were $\mathrm{CD} 11 \mathrm{c}^{+}$cells, and LPS stimulation markedly up-regulated the expression of CD80 and CD86 within the CD11 $\mathrm{c}^{+}$population (Figures 2A,D) and induced substantial release of IL-12 to the culture supernatants (Figure 2C), suggesting most cells harvested on day 6 were DCs. BA inclusion showed no effects on the upregulation of CD80/CD86 expression in response to the stimulation of LPS (Figures 2A,D), but resulted in a concentration-dependent reduction of total numbers of viable $\mathrm{CD} 11 \mathrm{c}^{+}$cells recovered on day 6 of culture (Figure 2B), and significantly suppressed the release of IL-12 to culture supernatants (Figure 2C). To examine whether $\mathrm{BA}$ reduced cell recovery by inducing cell death, apoptosis in the day-6 DCs was further assessed by FACS after annexin V/PI staining.
A significant degree of apoptosis was detected in the BA-treated DC, as shown by an increased phosphatidyl exposure using annexin V-FITC (Figure 2E).

\section{COMPARISON OF BA-INDUCED APOPTOSIS IN IMMATURE AND MATURE DC}

We further compared BA-induced apoptosis in mature DCs and immature DCs. In this experiment, the day-6 DCs stimulated with LPS were used as mature DCs, because they highly expressed CD80/ CD86 and secreted high level of IL-12. In comparison, the day-6 DCs without stimulation by LPS were used as immature DCs. Mature and immature DCs were treated with $50 \mu \mathrm{M}$ of BA for different time

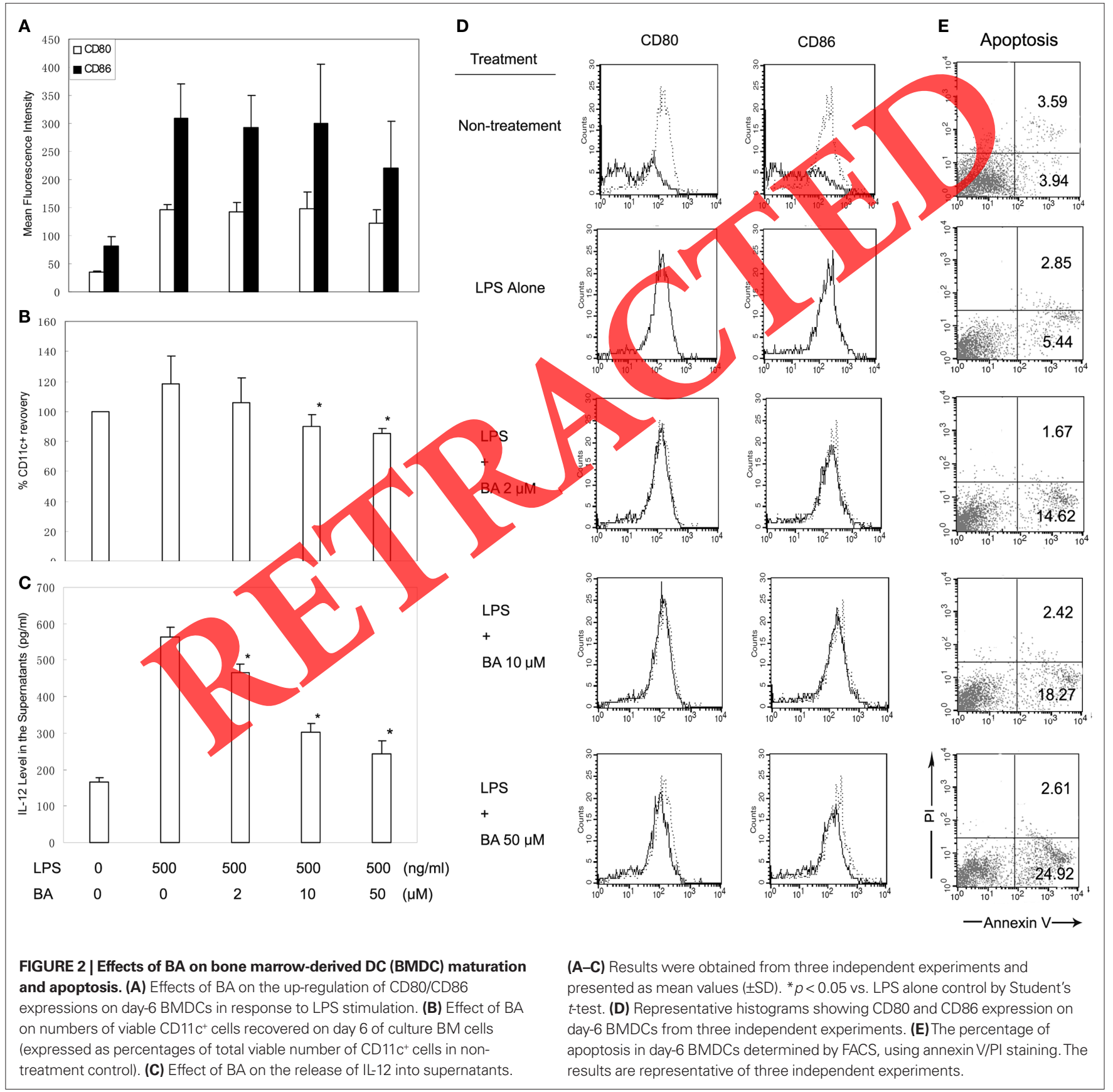




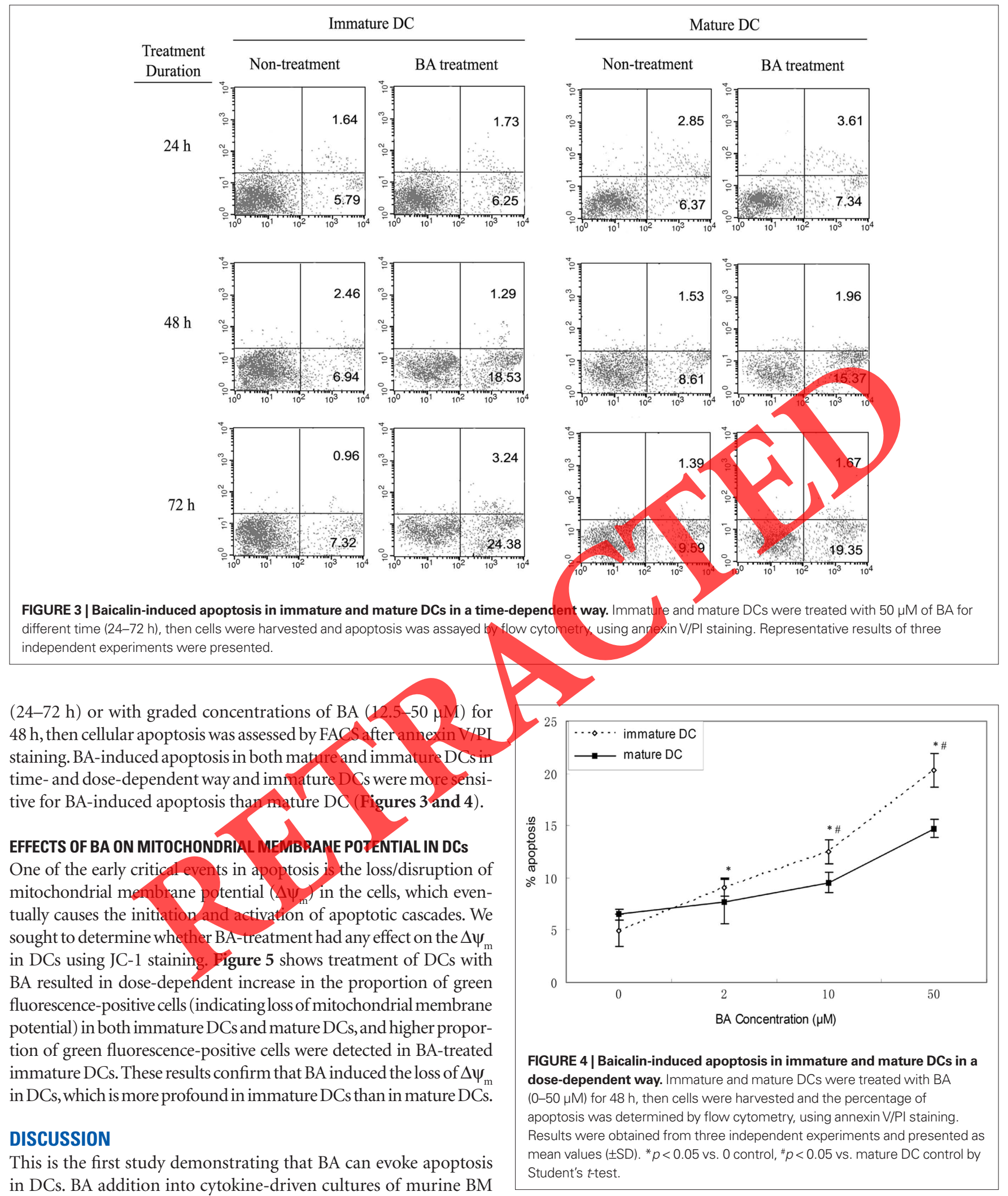

cells resulted in a reduced recovery of $\mathrm{CD} 11 \mathrm{c}^{+}$cells and less production of IL-12, but shown no effects on the up-regulation of CD80/ CD86 expression in response to the stimulation of LPS, suggesting BA has no influence on DC maturation, but may induce DC

apoptosis. The fact that BMDCs underwent strong apoptosis in the presence of BA in a time- and dose-dependent manner further confirmed its pro-apoptotic activity. Interestingly, immature DCs 


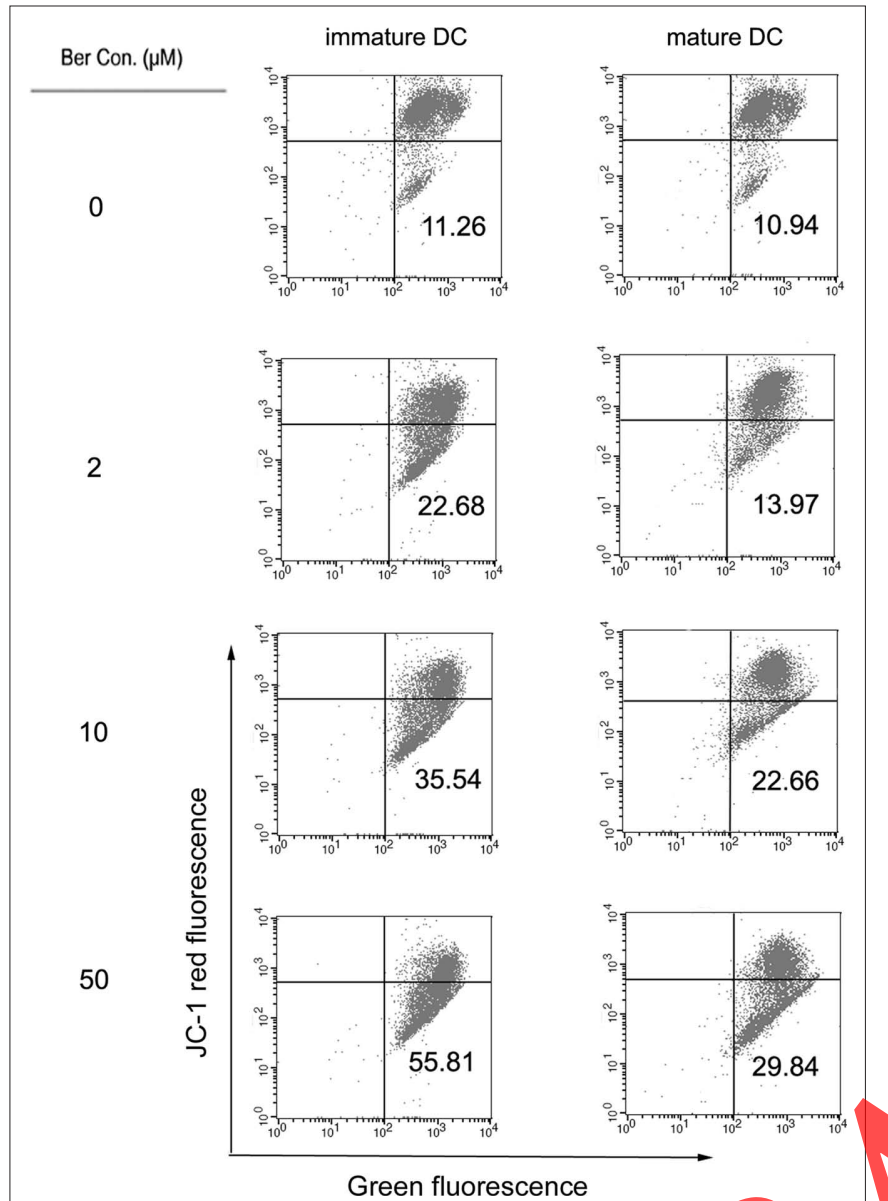

FIGURE 5 |The impact of BA on mitochondrial transmembrane potential in immature and mature DC. Immature and mature DCs were treated with BA $(0-50 \mu \mathrm{M})$ for $6 \mathrm{~h}$, stained with JC-1 dye probe, cytometry. Numbers in lower right quadrant indi that emit only green fluorescence which is attributed to depola mitochondrial membrane. Representative of three individual experiments with similar results are shown.

were found more sensitive for BA-induced apoptosis than mature DC. Concordantly, BA induced more profound change of $\Delta \psi_{\mathrm{m}}$ in immature DCs than in mature DCs, suggesting BA promote apoptosis through mitochondria-mediated pathway in DCs. A recent study investigated the effects of BA on apoptotic changes in multiple organs of severe acute pancreatitis rats. The results indicated that the apoptosis indexes significantly increased in lymph nodes and spleen in BA-treated group (Tian et al., 2009). Therefore, BA-induced DC apoptosis and the importance in vivo warrant further investigation.

Apoptosis regulates many aspects of immunologic homeostasis, including initiation, magnitude, and termination of immune responses (Giovannetti et al., 2008). During an immune response, homeostasis is disturbed as DCs become activated and promote the clonal expansion of antigen-specific lymphocytes. Shortly after the peak of the response, controlled induction of apoptosis, of both DCs and lymphocytes, restores homeostasis. This process is critical to ensure protective immunity and avoid lymphoid neoplasia and autoimmunity (Hildeman et al., 2007). While decreased apoptosis can cause an overabundance of lymphocytes and possibly autoimmune reactions, enhanced apoptosis can result in lymphocyte depletion and immunosuppression, so that the possibility to interfere with regulatory mechanisms of apoptosis, aiming either to block or to enhance it at different time points, represents a promising approach for the development of new immunomodulatory therapies (Rashedi et al., 2007). Previous studies have demonstrated that BA can inhibit the proliferation of T-lymphocytes (Li et al., 2009) and induce apoptosis in Jurkat T cells (Ueda et al., 2002). Here, we provide evidence that DCs may also primary target of BA for immunomodulation.

Traditionally, the development of immunosuppressive drugs and understanding of their action has been focused on lymphocytes. Many classical immunosuppressive agents, such as cyclosporine A, rapamycin, dexamethasone, and 1,25(OH)2D3, were found to modulate immune responses by inhibiting lymphocyte proliferation or promoting their apoptosis. However, recent evidence indicates that these agents interfere with immune responses at the earliest stage, targeting key functions of DCs (Hackstein and Thomson, 2004). For example, 1,25(OH)2D3 inhibits the differentiation and maturation of human DCs, abrogates the capacity of mature DCs to secrete IL-12 upon activation, while strongly enhancing IL-10 production, and promotes DC apoptosis (Penna

and Adorini, 2000). Rapamycin has also been reported to induce apoptosis specifically in DCs but not in monocytes/macrophages (Woltman et al., 2001). Dexamethasone selectively inhibits differentiation of cord blood stem cell derived-DC precursors into immature DCs and selectively induces apoptosis of developing DCs (Mainali and Tew, 2004). All of these studies clearly establish that DCs are one of primary targets of many immunosuppressive drugs.

Our data indicate that BA induces selective apoptosis in immature DCs possibly through mitochondria-mediated pathway, suggesting a novel mechanism of immunomodulation by BA which may account, at least in part, for the immunosuppressive effects observed in the animal models and in clinical settings. The fact that BA, next to the antiproliferative effect on $\mathrm{T}$ cells, selectively induces apoptosis in immature DCs suggests that BA may have potential applications as a supportive treatment for autoimmunemediated diseases.

\section{ACKNOWLEDGMENTS}

This work was partially supported by New Drug Innovation of the Ministry of Science and Technology, China (2009ZX09311011 ) and the National Natural Science Foundation of China (No 30725045, No 81072653).

\section{REFERENCES}

Banchereau, J., Briere, F., Caux, C., Davoust, J., Lebecque, S., Liu, Y. J., Pulendran, B., and Palucka, K. (2000). Immunobiology of dendritic cells. Annu. Rev. Immunol. 18, 767-811.
Banchereau, J., and Steinman, R. M. (1998). Dendritic cells and the control of immunity. Nature 392, 245-252.

Butcher, E. C., and Picker, L. J. (1996). Lymphocyte homing and homeostasis. Science 272, 60-66.
Chen, M., Wang, Y. H., Wang, Y., Huang, L., Sandoval, H., Liu, Y. J., and Wang, J. (2006). Dendritic cell apoptosis in the maintenance of immune tolerance. Science 311 , $1160-1164$.
Chung, C. P., Park, J. B., and Bae, K. H. (1995). Pharmacological effects of methanolic extract from the root of Scutellaria baicalensis and its flavonoids on human gingival fibroblast. Planta Med. 61, 150-153. 
Cossarizza, A., Baccarani-Contri, M., Kalashnikova, G., and Franceschi, C. (1993). A new method for the cytofluorimetric analysis of mitochondrial membrane potential using the J-aggregate forming lipophilic cation 5,5',6,6'-tetrachloro-1, $1^{\prime}, 3,3^{\prime}$ tetraethylbenzimidazolcarbocyanine iodide (JC-1). Biochem. Biophys. Res. Commun. 197, 40-45.

Giovannetti, A., Pierdominici, M., Di Iorio, A., Cianci, R., Murdaca, G., Puppo, F., Pandolfi, F., and Paganelli, R. (2008). Apoptosis in the homeostasis of the immune system and in human immune mediated diseases. Curr. Pharm. Des. 14, 253-268.

Hackstein, H., and Thomson, A. W. (2004). Dendritic cells: emerging pharmacological targets of immunosuppressive drugs. Nat. Rev. Immunol. 4, 24-34.

Hildeman, D., Jorgensen, T., Kappler, J., and Marrack, P. (2007). Apoptosis and the homeostatic control of immune responses. Curr. Opin. Immunol. 19, 516-521.

Huang, X., Tu, C. H. L., Zhang, L. X., Dai, J. R., and Jin, Y. D. (1986). Immunopharmacology. Shanghai: Shanghai Science and Technology Press.

Inaba, K., Inaba, M., Romani, N., Aya, H., Deguchi, M., Ikehara, S., Muramatsu, S., and Steinman, R. M. (1992). Generation of large numbers of dendritic cells from mouse bone marrow cultures supplemented with granulocyte/macrophage colonystimulating factor. J. Exp. Med. 176, 1693-1702.
Jung, S., Unutmaz, D., Wong, P., Sano, G., De los Santos, K., Sparwasser, T., Wu, S., Vuthoori, S., Ko, K., Zavala, F., Pamer, E. G., Littman, D. R., and Lang, R. A. (2002). In vivo depletion of CD11c+ dendritic cells abrogates priming of CD8+ T cells by exogenous cell-associated antigens. Immunity 17 , 211-220.

Koopman, G., Reutelingsperger,C.P., Kuijten, G.A., Keehnen, R. M., Pals, S. T., and van Oers, M. H. (1994). Annexin V for flow cytometric detection of phosphatidylserine expression on B cells undergoing apoptosis. Blood 84, 1415-1420.

Krakauer, T., Li, B. Q., and Young, H. A. (2001). The flavonoid baicalin inhibits superantigen-induced inflammatory cytokines and chemokines. FEBS Lett. 500, 52-55.

Kubo, M., Matsuda, H., Tanaka, M., Kimura, Y., Okuda, H., Higashino, M., Tani, T., Namba, K., and Arichi, S. (1984). Studies on Scutellariae radix. VII. Anti-arthritic and antiinflammatory actions of methanolic extract and flavonoid components from Scutellariae radix. Chem. Pharm. Bull. (Tokyo) 32, 2724-2729.

Li, L., Zeng, Y. Y., Huang, X. Y., Shong, B., Yang, Z., Teng, F., and Yao, M. L. (2009). Effects of baicalin on in vitro proliferation and cell cycle of murine T-lymphocytes. Chin. J. Cell. Mol Immunol. 25, 75-78.

Lin, C. C., and Shieh, D. E. (1996). The anti-inflammatory activity of Scutellaria rivularis extracts and its active components, baicalin, baicalein and wogo $m$. Chir 31-36.
Mainali, E. S., and Tew, J. G. (2004). Dexamethasone selectively inhibits differentiation of cord blood stem cell derived-dendritic cell (DC) precursors into immature DCs. Cell. Immunol. 232, 127-136.

Penna, G., and Adorini, L. (2000). 1 Alpha,25-dihydroxyvitamin D3 inhibits differentiation, maturation, activation, and survival of dendritic cells leading to impaired alloreactive $\mathrm{T}$ cell activation. J. Immunol. 164 2405-2411.

Rashedi, I., Panigrahi, S., Ezzati, P., Ghavami, S., and Los, M. (2007). Autoimmunity and apoptosis therapeutic implications. Curr. Med. Chem. 14, 3139-3151.

Tian, H., Zhang, X., Wu, C., Chen, L., Ying, R., Ye, J., Yu, B., Ye, Q., Pan, Y., Ma, M., and Zhu, F. (2009). Effects of baicalin and octreotide on the serum TNF-alpha level and apoptosis in multiple organs of rats with sever acute pancreatitis. Inflammation 32, 191-201.

Ueda, S., Nakamura, H., Masutani, Sasada, T., Takabayashi, A., Yamaoka, Y., and Yodoi, J. (2002) Baicalin induces apoptosis via mitochondrial pathway as prooxidant. Mol. Immunol. 38, 781-791.

Woltman, A. M., de Fijter, J. W., Kamerling, S. W., van Der Kooij, S. W., Paul, L. C., Daha, M. R, and van Kooten, C. (2001). Rapamycin induces apoptosis in monocyte- and CD34-derived dendritic cells but not in monocytes and macrophages. Blood 98, 174-180. eng, Y., Song, C., Ding, X., Ji, X., Yi, L. and Zhu, K. (2007). Baicalin reduces the severity of experimental autoimmune encephalomyelitis. Braz. J. Med. Biol. Res. 40, 1003-1010.

Zhang, M., Tang, H., Guo, Z., An, H., Zhu, X., Song, W., Guo, J., Huang, X., Chen, T., Wang, J., and Cao, X. (2004). Splenic stroma drives mature dendritic cells to differentiate into regulatory dendritic cells. Nat. Immunol. 5, 1124-1133.

Zhang, X. P., Tian, H., and Cheng, Q. (2003). The current situation in pharmacological study on baicalin. Chin. Pharmacol. Bull. 19, 1212-1215.

Conflict of Interest Statement: The authors declare that the research was conducted in the absence of any commercial or financial relationships that could be construed as a potential conflict of interest.

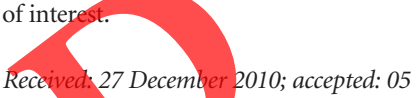
March 2011; published online: 29 March 2011.

Citation: Zhang H, Jiao Q, Gong Q, Zhang Y, Zhang W and Hu Z (2011) Baicalin induced dendritic cell apoptosis in vitro. nt. Pharmacol. 2:15. doi: 10.3389/ fphar.2011.00015

This article was submitted to Frontiers in Pharmacology of Natural Products, a specialty of Frontiers in Pharmacology.

Copyright (c) 2011 Zhang, Jiao, Gong, Zhang, Zhang and Hu. This is an openaccess article subject to an exclusive license agreement between the authors and Frontiers Media SA, which permits unrestricted use, distribution, and reproduction in any medium, provided the original authors and source are credited. 